\title{
Leaf Pubescence: The Significance of Lower Surface Hairs for the Spectral Properties of the Upper Surface
}

\author{
BENNO M. ELLER \\ Institute of Plant Biology, University of Zurich, Zollikerstrasse 107, CH 8008 Zurich, \\ Switzerland
}

Received 28 February 1977

\begin{abstract}
The fraction of the global radiation incident on the upper surface of leaves of Tussilago farfara L. which is reflected, varies according to the presence or absence of hairs on the lower surface. Calculations of the radiation fluxes at 550 and $1000 \mathrm{~nm}$ wavelengths prove that these differences arise from a partial reflection of the radiation emerging from the lower epidermis by the hair cover.
\end{abstract}

\section{INTRODUCTION}

One of the major problems of leaf ecology is the influence of pubescence on the radiation budget. A recent review by Johnson (1975) on the ecological significance of leaf pubescence reveals the lack of sufficient information to lay down a definitive theory on the interactions between leaf pubescence and radiative energy exchange. In general leaves covered with a hairy layer have a higher reflectivity and a lower absorptivity compared with hairless leaves as is shown by Shull (1929), Billings and Morris (1951), Birkebak and Birkebak (1964), Sinclair and Thomas (1970), Ehleringer, Bjoerkman, and Mooney (1976), and Eller and Willi (1977). A distinct ecological group among the leaves is formed by those with hairs restricted to the lower surface. Comparing the spectral properties of the upper and the lower surface of such leaves cannot give the real differences caused by pubescence, since the values for the upper surface differ markedly from that of the lower surface with the hairs removed as shown by Eller and Willi (1977). Experiments carried out recently by Eller and Willi (1977) with leaves of Tussilago farfara L. (coltsfoot) led to the supposition that the hairs on the lower surface of the leaves might increase the measured reflectivity of the upper surface. Additional research reported here verified this hypothesis.

\section{MATERIALS AND METHODS}

The diffuse reflectivity $(r)$ and the diffuse transmissivity $(t)$ for radiation incident on the upper surface were determined by means of a spectroradiometer ISCO SR with an integrating sphere of special design and a light source as described by Eller (1972). The reflectivity is defined as the quotient of the incident radiation and the fraction of this radiation which is measured as 
the radiation coming from the surface. The transmiseivity is the quotient of the incident radiation and that fraction of this radiation which emerges from the reverse side of the leaf. The ab. sorptivity $(a)$ is calculated from the equation

$$
a=1-(r+t) \text {. }
$$

Measurements were made for wavelengths from 400 to $1350 \mathrm{~nm}$. For comparison the hairs on the lower surface were removed by tearing them off with masking tape. After the measurements were carried out the epidermis of the lower surface was inspected with a scenning electron microscope to ascertein that the epidermis had not been damaged.

\section{RESULTS}

In Fig. 1 the spectral properties of the upper surface are shown for a hairy and a hairless lower surface. Within the wavelength range from 400 to $700 \mathrm{~nm}$ the values for the absorptivity differ only in the region of the green where absorptivity is at a relative minimum (Gates, Keegan, Schleter, and Weidner, 1965). For wavelengths from 700 to $1150 \mathrm{~nm}$ removing the hairs alters the reflectivity as well as the transmissivity. No effect is produced upon the absorptivity since these changes are of the same magnitude and opposite in direction. For wavelengths beyond $1150 \mathrm{~nm}$ a slight increase of the absorptivity is observed for the leaf with hairs on the lower surface.

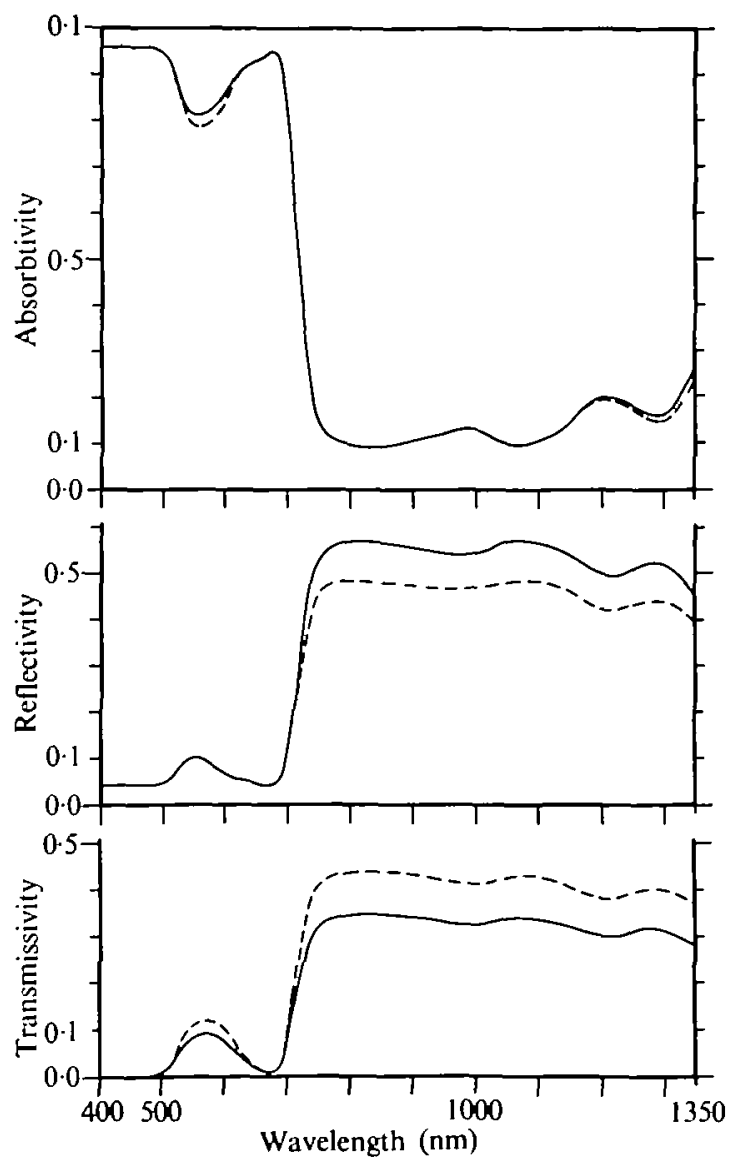

F1G. 1. Spectral properties of the upper surface of an adult leaf of Tussilago farfara L. with hairs on the lower surface (-) and the hairs removed (--- ). 
It can be assumed that the hairs on the lower surface act as a reflecting layer. In the presence of hairs the transmitted radiation emerging from the lower epidermis is partially reflected back to the leaf and the remainder is the transmitted radiation. Some minor part of this radiation might be absorbed by the hairs. The radiation reflected back into the leaf makes its way once more through the different leaf tissues. Depending on whether or not the absorptivity at a specific wavelength is high or low, a major or a minor part of this reflected radiation is absorbed while the rest emerges from the upper epidermis. The latter part of the radiation will increase the amount of the radiation coming from the upper surface and which is measured as the so-called reflected radiation. The directly reflected radiation from the upper surface cannot be detected separately from the radiation which leaves the epidermis after a two-fold transmission through the leaf following a reflection at the hairs on the lower surface.

As shown by Eller and Willi (1977) the spectral reflectivities of the lower surface are almost uniformly increased by hairs within the wave range observed. A wavelength-independent reflection power of the white hair felt can be assumed and a uniform influence on the measured spectral reflectivity of the upper surface might be suggested. This is evidently not the case as is shown by Fig. 1. The reasons why no changes can be detected on the upper surface 'reflectivity' up to a wavelength of about $700 \mathrm{~nm}$ are (1) because the values for the transmitted radiation within that wave range are very low and (2) that the small amount of radiation which could be reflected by the hair cover on the lower surface is absorbed on its way back through the leaf. Furthermore, fractions below $0.5 \%$ of the incident radiation cannot be detected by the equipment used for the measurements. If the amount of the transmitted radiation increases, more radiation is reflected by the hair cover and thus the changes become measurable. Alterations in the spectral properties which are derived from these radiation measurements can then be observed, as can be seen for the wavelengths at about $550 \mathrm{~nm}$ and beyond $1150 \mathrm{~nm}$. The fraction of the radiation transmitted by a hairless leaf reflected by the hair cover can be estimated by checking the differences for the transmission in the presence and the absence of lower surface hairs.

At wavelengths from 700 to $1150 \mathrm{~nm}$ no changes in the absorptivity are detectable since the absorptivities of the leaf are very low. Hence nearly the whole radiation reflected by the hairs on the lower surface emerges from the upper epidermis and increases the values for the reflectivities of the upper surface.

\section{MODEL OF RADIATION FLUXES AND CONCLUSIONS}

A model of the radiation fluxes at and through the leaf is shown in the lower part of Fig. 2. The incident radiation $(I)$ is partly reflected $\left(R_{\mathrm{N}}\right)$ at the upper surface with the reflectivity $r_{\mathrm{N}}$ measured without hairs on the lower surface. The remainder $\left(I_{\mathrm{L}}\right)$ of the incident radiation penetrates into the leaf and is partly absorbed $\left(A_{\mathrm{N}}\right)$ with the absorptivity $a_{N}$ measured without hairs on the lower surface and the rest $\left(I_{\mathbf{H}}\right)$ forms the input into the hair layer. If the hairs are removed this radiation $I_{\mathrm{H}}$ is the radiation $T_{\mathrm{N}}$ transmitted by the leaf. For the intact leaf with hairs, this radiation is split up into the radiation $\left(I_{\mathbf{L L}}\right)$ reflected back to the leaf and the radiation transmitted $\left(T_{\mathrm{H}}\right)$. The radiation $I_{\mathrm{LL}}$ penetrates into the leaf and on its way through the leaf 

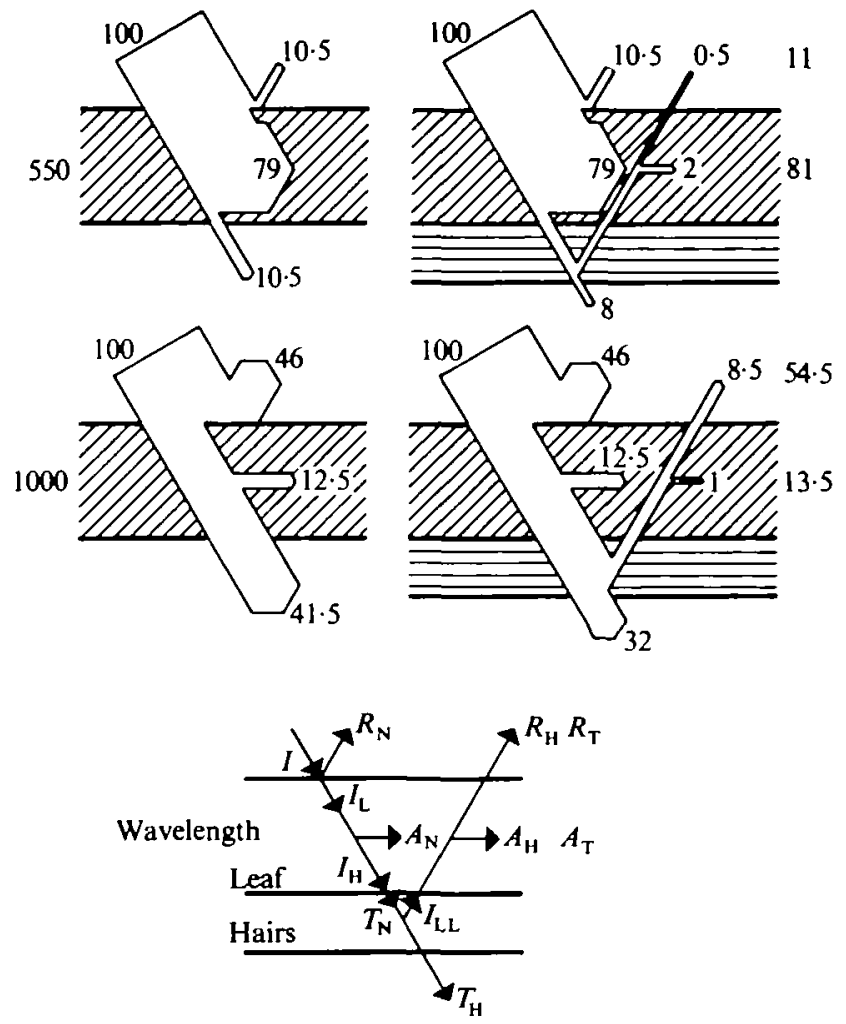

Fia. 2. Leaf model for Tussilago farfara L. with hairs on the lower surface (right) and the hairs removed (left). Radiation flures at 550 and $1000 \mathrm{~nm}$ wavelength. $I$, incident radiation; $R_{\mathrm{N}}$, reflected radiation without hairs; $I_{\mathrm{L}}$, radiation penetrating into the leaf; $A_{\mathrm{N}}$, absorbed and $T_{\mathrm{N}}$, tranamitted radiation without hairs; $I_{\mathrm{B}}$, radiation emerging from the lower epidermis; $I_{\mathrm{LL}}$, radiation reflected by the hair cover; $T_{\mathrm{H}}$, radiation transmitted by the hairy leaf; $R_{\mathrm{T}}$, total of the reflected and $A_{\mathrm{T}}$, trotal of the absorbed rad lation; $R_{\mathrm{H}}$, additional radiation emerging from the upper epidermis; $A_{\mathrm{H}}$, absorbed fraction of the reflected radiation $I_{\mathrm{LL}}$.

tissues a part $\left(A_{\mathrm{H}}\right)$ is absorbed with the absorptivity value $a_{\mathrm{L}}$ measured for the hairless lower surface. The remainder forms the radiation $R_{\mathrm{H}}$ emerging from the upper surface. The absorbed and the reflected radiation for the leaf with hairs are $A_{\mathrm{T}}$ and $R_{\mathrm{T}}$ respectively.

Only the spectral properties and the radiation fluxes of both surfaces with hairs or hairless are measurable. The values of $I_{\mathrm{LL}}, A_{\mathrm{H}}$, and $R_{\mathrm{H}}$ are determinable only by calculations. To verify the influence of the hair-covered lower surface upon the measured reflectivity of the upper surface, calculations of the splitting of the incident radiation into the different radiation fluxes can be made. The wavelengths at which such calculations should be performed are 550 and $1000 \mathrm{~nm}$ respectively. At $550 \mathrm{~nm}$ the absorptivity of the leaf is high and a difference between its values with a hairy or a hairless lower surface is detectable. At $1000 \mathrm{~nm}$ no differences are present and the absorptivity of the leaf is low. The equations to calculate the values not measurable are

$$
\begin{gathered}
A_{\mathrm{N}}=I-\left(R_{\mathrm{N}}+T_{\mathrm{N}}\right) \\
I_{\mathrm{LL}}=T_{\mathrm{N}}-T_{\mathrm{H}}
\end{gathered}
$$




$$
\begin{gathered}
A_{\mathrm{H}}=I_{\mathrm{LL}} \cdot a_{\mathrm{L}} \\
R_{\mathrm{H}}=I_{\mathrm{LL}}-A_{\mathrm{H}} \\
A_{\mathrm{T}}=A_{\mathrm{N}}+A_{\mathrm{H}} \\
R_{\mathrm{T}}=R_{\mathrm{N}}+R_{\mathrm{H}} \\
A_{\mathrm{T}}=I-\left(R_{\mathrm{T}}+T_{\mathrm{H}}\right) .
\end{gathered}
$$

As shown in a detailed review of the spectral properties of juvenile and adult leaves of Tussilago farfara L. by Eller and Willi (1977) the values for $a_{\mathrm{N}}$ and $a_{\mathrm{L}}$ at the wavelengths selected for the calculations are nearly equal and the value of $a_{N}$ can be taken for $a_{\mathrm{L}}$ to carry out the calculations. The values for the reflected radiation and for the reflectivity expressed as percentages are the same if a value of 100 is taken for the incident radiation. The same is valid for the transmitted radiation and the transmissivity and also for the absorbed radiation and the absorptivity.

On the left of Fig. 2 the measured values for the leaf without hairs on the lower surface are shown. Substituting these values in the equations (2) to (7) we can calculate the radiative fluxes which cannot be measured in a direct way. On the right of Fig. 2 the measured and the calculated values are displayed for the leaf with hairs. The absorptivity $A_{T}$ calculated with the aid of equation (6) is the same as might be derived from measurements and using equation (8). The calculated reflectivity $R_{\mathrm{T}}$ is equal to the measured reflectivity. These coinciding values give the proof that the differences for the reflectivity of leaves with hairs present or absent from the lower surface arise from the reflection of a part of the radiation by the hair layer. Furthermore, they are affected by a more or less strong absorption of this radiation on its return through the leaf. If the absorptivity is high, as it is at $550 \mathrm{~nm}$ wavelength, then only a small fraction of that reflected radiation contributes to an increase of the radiation coming from the upper surface as 'reflected' radiation. At $1000 \mathrm{~nm}$ wavelength the absorptivity is low and therefore a much greater increase of the 'reflectivity' of the upper surface is the result. The same relations might be shown for every wavelength.

It must be emphasized that hairs on the lower surface do not affect the absorptivity to an extent that could be significant for the determination of the amount of absorbed energy of global radiation.

Moreover, this work shows the extent to which the measured values of the reflectivity might be miscalculated if insufficient care is taken to avoid any direct incident radiation or reflected radiation falling on the reversed surface of the leaf when the reflected radiation of the upper surface is measured. Perhaps some of the variable results on the reflectivities of leaves have been caused by such stray light.

\section{ACKNOWLEDGEMENTS}

This research work was supported by the Swiss National Foundation grant 3.745.72. I thank Dr. R. Parish for his help in the preparation of the manuscript. 
LITERATURE CITED

BILLIngs, W. D., and Morris, R. J., 1951. Am. J. Bot. 38, 327-31.

BIRKEBAK, Roland, and BIRKEBAK, Riohard, 1964. Ecology, 45, 646-9.

EhLERINGer, J., BJokrkoman, O., and Mooney, H. D., 1976. Science, N.Y. 192, 376-7.

ElLkir, B. M., 1972. Verh. Schweiz. Natf. Ges. 152, 142-5.

and WrifI, P., 1977. Oecologia 25, 179-87.

Gates, D. M., KegaAN, H. J., SoHLETER, J. C. and WeIONER, V. R., 1965. Appl. Opt. 4, 11-20.

JoHNgon, H. B., 1975. Bot. Rev. 41, 233-58.

Shull, Ch. A., 1929. Bot. Gaz. 87, 583-607.

Sinctatr, R., and Thomas, D. A., 1970. Aust. J. Bot. 18, $261-73$. 